\title{
Dietary Patterns in High and Low Consumers of Meat in a Swedish Cohort Study
}

\section{S. ELMSTÅHL}

Department of Community Medicine, Lund University

\section{O. HOLMOVIST}

Swedish Meat Research Institute

B. GULLBERG

Department of Community Medicine, Lund University

\author{
U. JOHANSSON and G. BERGLUND \\ Department of Medicine, Lund University
}

\begin{abstract}
The objective was to examine relationships between meat and other food items which have been associated with higher risk of cancer in the colon and prostate in some epidemiological studies. The study was conducted as a population-based cohort study comprising 11648 subjects (4816 male and 6742 female) born between 1926 and 1945 and living in the city of Malmö, Sweden. Data on mean daily intake of foods and nutrients were assessed with a diet history method combining a 7-day menu book and a food frequency questionnaire. Increasing meat intake, expressed in quintiles and adjusted for energy, was associated with decreasing intakes of poultry, fish, fruits, bread, cereals and cheese in both sexes. Low negative correlations between meat intake and ascorbic acid $(r=-0 \cdot 11)$ and fiber $(r=-0 \cdot 16$ to $-0 \cdot 20)$ were noted. The average intake of fat from meat out of total fat intake was $13.6 \%$ in men and $11.9 \%$ in women. No major associations were noted between meat and the cholesterol raising fatty acids $\mathrm{C}: 12: 0, \mathrm{C}: 14: 0$, C:160 nor for C:20:4 or its precursor C:18:2.

In conclusion, our findings indicate that meat consumption is negatively associated with food groups rich in antioxidants and fiber and the positive covariance reported between meat and cancer and coronary heart disease might, therefore, not be directly linked to components in meat.
\end{abstract}

(c) 1999 Academic Press

\section{INTRODUCTION}

So called "diseases of affluence", such as certain cancers and cardiovascular diseases, are prevalent in countries with a diet characterized by a high consumption

This study was supported by the Swedish Cancer Society (2684-B93-05XAA) and the Swedish Meat Information Institute.

Address correspondence to: Sölve Elmståhl, Dept of Community Medicine, Malmö University Hospital, S-205 02 Malmö, Sweden. 
of meat and dairy products, often rich in saturated fat and sugar rich foods. Several epidemiological studies on diet and cancer have suggested associations between per capita consumption of fat intake and cancers of the large bowel, breast and prostate (Armstrong \& Doll, 1973; Carrol, 1975). However, different case-control studies and prospective studies have reported inconsistent results, although about 20 epidemiological studies have found associations between intake of fat and meat and colon cancer (Ewertz \& Gill, 1990; Miller et al., 1983; Potter, 1996). Out of six previous prospective studies on meat consumption and colon cancer, one Japanese study showed a decreased risk (Hirayama, 1981), three studies - the study of Sevenday Adventists (Philips \& Snowdon, 1985), the IOWA women study (Bostick et al., 1994) and a Dutch study (Goldbohm et al., 1994) - could not find any association, and two studies showed a positive association; the Nurses' Health study (Willett et al., 1990) and the Health Professionals study (Giovanucci et al., 1994). Another prospective study has demonstrated an association between meat and prostate cancer (Giovanucci et al., 1993).

Comparisons between studies are complicated by the use of different dietary assessment methods. Furthermore, risk models often include several variables, and observed relative risk estimates may be severely biased if these are intercorrelated (Elmståhl \& Gullberg, 1997). Since the same food item might comprise both carcinogenic substances and anti-carcinogens (e.g. antioxidants and protease inhibitors) collinearity between different nutrients might blur interpretations. Therefore, it seems important to study dietary habits and associations between meat intake and other foods. Intake of meat and meat products is related not only to other kinds of foods, but possibly also to other non-dietary lifestyle factors in a very complex way. Varying hypotheses and pathophysiological pathways have been suggested such as increased oxidative burden through a high intake of either iron or dietary fat which might follow a high meat intake. Several other different mechanisms have been proposed whereby dietary fat, especially animal fat, could increase risk of cancer in the large bowel, breast and prostate including associations between high fat intake and sex hormones and a cancer promoting effect of bile acids (Hill et al., 1987). As for cardiovascular diseases, meat can be implicated as a risk factor in at least three ways. Firstly, about half of the fat from meat is saturated fat, of which some saturated fatty acids presumably raise serum cholesterol in populations at risk for coronary heart disease (CHD) (Gotto et al., 1990). Secondly, meat provides arachidonic acid both directly and indirectly as a metabolite from linoleic acid. Arachidonic acid is a precursor of thromboxane $\mathrm{A}_{2}$ which increases platelet aggregation propensity (Galli et al., 1989; Leaf \& Weber, 1988). Thirdly, meat provides haeme iron which has been shown to promote oxidation of LDL in vitro (Balla et al., 1991). On the other hand, ecological study on CHD mortality and annual meat consumption in European countries showed no relationship; e.g. France with the highest meat consumption had the lowest CHD mortality rate (Ulbricht \& Southgate, 1991).

The specific aim of this study was to assess meat consumption in relation to intake of nutrients, e.g. the intake of energy, fat, fatty acids and micronutrients and intakes of other foods, e.g. vegetables, fruits, dairy products and cereals. This will provide a background for more valid interpretation of forthcoming results from the ongoing Malmö Diet and Cancer Study on the relationship between meat intake and disease. 


\section{Materials AND Methods}

This study emanates from the Malmö Diet and Cancer study, planned and conducted as a prospective cohort study. The general outline and design of the cohort study has been reported elsewhere (Berglund et al., 1993). The study was planned involving subjects born between 1926 and 1945 in the municipality of Malmö, a city in southern Sweden with about 240000 inhabitants. Food intake, socio-economic factors, lifestyle pattern, current diseases, symptoms and medication were determined and incidence of cancer will be followed for 10 years by registers. Subjects with insufficient understanding of the Swedish language were excluded. The study population, 11646 subjects, was representative of all age groups born between 1926 and 1945, out of all 53000 subjects at the same age, in the municipality of Malmö (in February 1991). Somewhat more women than men were recruited, see Table 1. Sixty-eight percent of all subjects were married, 17\% divorced, $9 \%$ single and $6 \%$ widowed. Approximately $15 \%$ of the cohort had a foreign background (Swedish citizen, but born abroad) or were foreign citizens.

The study population comprised 11646 subjects, (4858 male and 6788 female) who fulfilled the diet assessment and questionnaires during 1991-1993. Permanent residents in the city of Malmö were randomly invited to participate by letter and by advertisements in the local media. The information on place of residence was obtained from the city Council Population Registry and updated every third month.

The diet assessment method used in this study is a modified diet history method, combining a 7-day menu book covering hot meals and beverages, and a quantitative food frequency questionnaire for other meals covering breakfast, snacks and fruits over the past year. In order to cover seasonal variation, intake of different kinds of fruits were asked for different seasons separately. In both parts, the usual portion sizes of each of the different food items and dishes are estimated with the help of a picture book with approximately 180 photographs of varying portions of different types of foods. Mean daily intake of the food items was calculated. The participants visted the Study centre twice. At the first visit, the participants were given information about background and objectives of the study in groups of six to eight people. The questionnaires and dietary assessment booklets were introduced and explained. During the second visit the completeness of the questionnaires was checked individually by a diet assistant. Average serving sizes of each food item written in the menu book were determined with the help of the picture book. No compensation was made for losses of fat from meat and meat products during frying or boiling, except for bacon. However, added fat during frying was not included, except when the meat was prepared with a coating. The data were entered directly into a computer program and then checked for abnormal or missing values. Measured energy intake was also compared to estimated energy expenditure adjusted for age, gender and weight. The lowest and highest percentiles of calculated differences between individual energy intake and energy expenditure, according to the $1985 \mathrm{FAO} / \mathrm{WHO} / \mathrm{UNU}$ report on energy requirements, were controlled separately. The nutrient values of the estimated food intake were calculated using the Swedish Food Database from the Swedish National Food Administration. The method allows the calculation of intake of micro- and macronutrients, different foods and dishes, and the intake of nutrients per different food groups. The consumption of dietary supplements was recorded in the 7-day menu book and the contribution of nutrients from supplements 
TABLE 1

Distribution of age, gender and marital status in the study population and the total Malmö Diet and Cancer Study (MDC) cohort number and percentage)

\begin{tabular}{|c|c|c|c|c|c|c|}
\hline & \multicolumn{2}{|c|}{ Men } & \multicolumn{2}{|c|}{ Women } & \multicolumn{2}{|c|}{ Total } \\
\hline & $\begin{array}{l}\text { Participants } \\
(N=4858)\end{array}$ & $\begin{array}{c}\text { MDC } \\
(N=25527)\end{array}$ & $\begin{array}{l}\text { Participants } \\
(N=6788)\end{array}$ & $\begin{array}{c}\text { MDC } \\
(N=27473)\end{array}$ & $\begin{array}{l}\text { Participants } \\
(N=11646)\end{array}$ & $\begin{array}{c}\text { MDC } \\
(N=53000)\end{array}$ \\
\hline \multicolumn{7}{|l|}{ Year of birth } \\
\hline 1962-30 & $29 \cdot 9$ & $24 \cdot 5$ & $28 \cdot 1$ & $26 \cdot 6$ & $28 \cdot 8$ & $25 \cdot 6$ \\
\hline $1931-35$ & $23 \cdot 3$ & $22 \cdot 9$ & $23 \cdot 5$ & $23 \cdot 2$ & $23 \cdot 4$ & $23 \cdot 0$ \\
\hline $1936-40$ & $22 \cdot 3$ & $24 \cdot 0$ & $23 \cdot 7$ & $23 \cdot 6$ & $23 \cdot 1$ & $23 \cdot 8$ \\
\hline $1941-45$ & $24 \cdot 5$ & $28 \cdot 6$ & $24 \cdot 7$ & $26 \cdot 6$ & $24 \cdot 6$ & $27 \cdot 6$ \\
\hline Gender & $41 \cdot 3$ & $48 \cdot 2$ & $58 \cdot 7$ & $51 \cdot 8$ & - & - \\
\hline \multicolumn{7}{|c|}{ Marital status } \\
\hline Unmarried & $10 \cdot 3$ & $13 \cdot 0$ & $8 \cdot 1$ & $8 \cdot 7$ & $8 \cdot 9$ & $10 \cdot 8$ \\
\hline Married & $74 \cdot 4$ & $62 \cdot 8$ & $64 \cdot 6$ & $57 \cdot 7$ & $68 \cdot 0$ & $60 \cdot 1$ \\
\hline Divorced & $13 \cdot 2$ & $19 \cdot 5$ & $19 \cdot 0$ & $23 \cdot 2$ & $16 \cdot 4$ & $21 \cdot 3$ \\
\hline Widowed & $2 \cdot 1$ & $2 \cdot 3$ & $8 \cdot 4$ & $9 \cdot 1$ & $5 \cdot 7$ & $5 \cdot 8$ \\
\hline
\end{tabular}


and their relationships to dietary habits have been described elsewhere (Elmståhl et al., 1994).

The method has been validated for nutrients as well as food items, using an 18day weighed dietary record as a reference, in a separate methodological study (Elmståhl et al., 1996a; Riboli et al., 1997). The results showed good agreement with the reference method for energy, macronutrients, minerals and vitamins with correlation coefficients in the order of $0 \cdot 6-0 \cdot 7$ and for the main 23 food groups covering habitual dietary habits in the order of $0 \cdot 5-0 \cdot 7$ using correlation analyses. Men tended to overestimate meat intake by $15 \%$, whereas women underestimated meat intake by 4\% (Elmståhl et al., 1996a). Only marginal differences were noted when crude or energy adjusted correlation coefficients were calculated for men and women. Corresponding study of reproducibility showed correlation coefficients between 0.6 and $0 \cdot 8$ for the majority of food groups (Elmståhl et al., 1996b).

\section{Statistical Analyses}

Meat consumers have been defined according to intake of meat, see definition below. Intake of specific food items separately, or from recipes, have been aggregated and intake calculated in grams using the Swedish Food Database, from the National Food Administration, comprising approximately 1500 food items. Meat consumers have been classified into quintiles according to meat intake, after adjustment for energy intake, using a residual regression model with energy as the dependent variable. Log transformed values were used to correct for skewness. Energy adjustment was performed to enable the relative contribution of meat to consumption of other foods to be studied independently of energy intake. High and low consumers of meat were defined as the population in the highest and lowest quintiles. A linear trend was tested between quintiles using Pearson product moment correlation. Association between food groups were analysed with Spearman rank correlation. All tests were two-sided. To calculate the consumption of food groups the prepared dishes were broken down to food items and all amounts of food belonging to the same food group were added, e.g. whole meat included in prepared dishes i.e. casserole or pie were added together. Meat comprises the sum of whole and processed meat. Whole meat includes whole and minced pork, beef, lamb and game and minced meat products, expressed as weight before cooking. Processed meat comprises sausages made from pork, beef, lamb and game and poultry, expressed as weight before cooking. Weight losses during processing is not compensated for. Intake of specific nutrients were calculated per intake of meat in grams.

\section{RESULTS}

Interrelations between different meat components and some other major food groups, adjusted for energy, are given in Table 2. Low correlations, in the order of $0 \cdot 0-0 \cdot 3$, were found between meat and other food groups for both men and women. Consumption of processed meat, comprising sausages from different meat sources, showed little or no correlation to intake of whole meat. The median daily intakes of meat were $104 \mathrm{~g}$ for men and $70 \mathrm{~g}$ for women, similar to the energy-adjusted values. 
TABLE 2

Spearman correlation coefficients between some major food groups in 4858 men and 6788 women, adjusted for energy. Data on women are shown in italics

\begin{tabular}{|c|c|c|c|c|c|c|c|c|}
\hline & Meat & $\begin{array}{l}\text { Whole } \\
\text { meat }\end{array}$ & $\begin{array}{c}\text { Processed } \\
\text { meat }\end{array}$ & Poultry & Vegetables & Potatoes & Fruits & $\begin{array}{l}\text { White } \\
\text { bread }\end{array}$ \\
\hline Meat & - & $0 \cdot 88$ & $0 \cdot 41$ & $-0 \cdot 12$ & -0.03 & $0 \cdot 28$ & $-0 \cdot 11$ & -0.06 \\
\hline Whole meat & $0 \cdot 86$ & - & $0 \cdot 11$ & $-0 \cdot 10$ & 0.01 & $0 \cdot 25$ & -0.08 & $-0 \cdot 05$ \\
\hline Processed meat & $0 \cdot 40$ & -0.03 & - & $-0 \cdot 05$ & -0.09 & $0 \cdot 15$ & -0.09 & $-0 \cdot 03$ \\
\hline Poultry & $-0 \cdot 12$ & -0.09 & -0.06 & - & $0 \cdot 06$ & -0.03 & $0 \cdot 04$ & $-0 \cdot 04$ \\
\hline Vegetables & $0 \cdot 15$ & $0 \cdot 06$ & $-0 \cdot 08$ & $0 \cdot 08$ & - & -0.05 & $0 \cdot 33$ & $-0 \cdot 12$ \\
\hline Potatoes & $0 \cdot 29$ & $0 \cdot 27$ & $0 \cdot 13$ & $0 \cdot 01$ & $0 \cdot 01$ & - & -0.09 & $0 \cdot 00$ \\
\hline Fruits & $-0 \cdot 14$ & $-0 \cdot 11$ & $-0 \cdot 07$ & $0 \cdot 04$ & $0 \cdot 27$ & $-0 \cdot 10$ & - & $-0 \cdot 13$ \\
\hline White bread & $-0 \cdot 14$ & $-0 \cdot 12$ & -0.07 & $-0 \cdot 01$ & -0.07 & -0.05 & $-0 \cdot 07$ & - \\
\hline
\end{tabular}


TABLE 3

Daily median intake of food groups in gram by quintiles of energy adjusted meat consumption in 4858 men

\begin{tabular}{|c|c|c|c|c|c|c|}
\hline \multicolumn{7}{|c|}{ Quintiles } \\
\hline $\begin{array}{l}\text { Food groups } \\
\text { median g/day }\end{array}$ & $\begin{array}{c}1 \\
\text { (Low) }\end{array}$ & 2 & 3 & 4 & $\begin{array}{c}5 \\
\text { (High) }\end{array}$ & $r$ \\
\hline Meat & 50 & 81 & 103 & 131 & 179 & - \\
\hline Fat content $\leq 10 \%$ & 15 & 28 & 37 & 46 & 64 & $0 \cdot 50^{\mathrm{a}}$ \\
\hline Fat content $>10 \%$ & 1 & 11 & 17 & 24 & 34 & $0 \cdot 39^{\mathrm{a}}$ \\
\hline Whole meat & 42 & 65 & 82 & 106 & 143 & $0 \cdot 72^{\mathrm{a}}$ \\
\hline Processed meat & 0 & 16 & 18 & 24 & 32 & $0 \cdot 38^{\mathrm{a}}$ \\
\hline Poultry & 17 & 9 & 1 & 0 & 0 & $-0 \cdot 14^{\mathrm{a}}$ \\
\hline Fish & 53 & 46 & 42 & 38 & 34 & $-0 \cdot 15^{\mathrm{a}}$ \\
\hline Potatoes & 111 & 133 & 144 & 152 & 168 & $0 \cdot 22^{\mathrm{a}}$ \\
\hline Fried & 0 & 0 & 0 & 0 & 0 & $0 \cdot 13^{\mathrm{a}}$ \\
\hline Boiled & 81 & 103 & 105 & 110 & 125 & $0 \cdot 17^{\mathrm{a}}$ \\
\hline Cereals & 29 & 25 & 22 & 21 & 18 & $-0 \cdot 15^{\mathrm{a}}$ \\
\hline Vegetables & 127 & 122 & 120 & 126 & 127 & $-0 \cdot 01$ \\
\hline Fruits & 175 & 164 & 154 & 143 & 135 & $-0 \cdot 13^{\mathrm{a}}$ \\
\hline Bread, white & 140 & 138 & 127 & 122 & 106 & $-0 \cdot 15^{\mathrm{a}}$ \\
\hline Bread, crisp & 18 & 15 & 15 & 15 & 13 & $-0 \cdot 09^{\mathrm{a}}$ \\
\hline Egg & 20 & 21 & 20 & 21 & 23 & $0.06^{\mathrm{a}}$ \\
\hline Milk & 260 & 289 & 266 & 252 & 226 & $-0.05^{\mathrm{a}}$ \\
\hline Cream & 11 & 12 & 11 & 11 & 10 & $0 \cdot 05^{\mathrm{a}}$ \\
\hline Cheese & 45 & 42 & 41 & 40 & 34 & $-0 \cdot 15^{\mathrm{a}}$ \\
\hline Edible fats & 51 & 53 & 51 & 48 & 44 & $-0 \cdot 10^{\mathrm{a}}$ \\
\hline Edible fat $100 \%$ & 0 & 0 & 0 & 0 & 0 & $-0.03^{\mathrm{c}}$ \\
\hline Edible fat $80 \%$ & 7 & 9 & 8 & 9 & 9 & $0.04^{c}$ \\
\hline Edible fat $60 \%$ & 0 & 0 & 0 & 0 & 0 & $0 \cdot 01$ \\
\hline Edible fat $40 \%$ & 14 & 21 & 17 & 15 & 12 & $-0 \cdot 07^{\mathrm{a}}$ \\
\hline Butter & 0 & 0 & 0 & 0 & 0 & $-0 \cdot 06^{\mathrm{a}}$ \\
\hline Mayonnaise/dressing & 2 & 3 & 2 & 3 & 2 & $0 \cdot 00$ \\
\hline Sweets & 20 & 19 & 19 & 18 & 15 & $-0.07^{\mathrm{a}}$ \\
\hline \multicolumn{7}{|l|}{ Alcoholic beverages } \\
\hline Hard liquor & 0 & 0 & 0 & 6 & 6 & $0 \cdot 08^{\mathrm{a}}$ \\
\hline Wine & 0 & 0 & 14 & 17 & 6 & $0 \cdot 02$ \\
\hline Beer & 0 & 43 & 29 & 29 & 29 & -0.02 \\
\hline
\end{tabular}

A significant trend between quintiles are given as Pearson correlation coefficients; ${ }^{\text {a }}$ denotes $p<0 \cdot 001$ and ${ }^{\mathrm{c}}=p<0 \cdot 05$

Meat consumption, adjusted for energy intake, was divided into quintiles. The age distributions were similar in all quintiles and the mean age varied between 55.9 and 57.7 years in men and between 55.6 and 57.2 years in women. The intake of different food groups in relation to meat consumption are given in Tables 3 and 4 for both sexes. Both men and women showed a decreasing intake of fish, poultry, cereals, bread, fruits, cheese and edible fats with increasing intake of meat. The intake of potatoes, and milk for women only, increased with higher intake of meat. Intake of alcoholic beverages in women was similar irrespective of meat intake, while in men the intake of hard liquor increased with increasing meat intake.

The intake of different nutrients in relation to meat consumption are shown for men and women in Tables 5 and 6 . High consumers of meat had higher intake of 
TABLE 4

Daily median intake of food groups in gram by quintiles of energy adjusted meat consumption in 6788 women

\begin{tabular}{|c|c|c|c|c|c|c|}
\hline \multicolumn{7}{|c|}{ Quantiles } \\
\hline $\begin{array}{l}\text { Food groups } \\
\text { median g/day }\end{array}$ & $\begin{array}{c}1 \\
\text { (Low) }\end{array}$ & 2 & 3 & 4 & $\begin{array}{c}5 \\
\text { (High) }\end{array}$ & $r$ \\
\hline Meat & 29 & 53 & 70 & 90 & 122 & - \\
\hline Fat content $\leq 10 \%$ & 7 & 20 & 27 & 32 & 45 & $0 \cdot 51^{\mathrm{a}}$ \\
\hline Fat content $>10 \%$ & 0 & 4 & 8 & 15 & 27 & $0 \cdot 44^{\mathrm{a}}$ \\
\hline Whole meat & 23 & 45 & 58 & 74 & 101 & $0 \cdot 78^{\mathrm{a}}$ \\
\hline Processed meat & 0 & 6 & 12 & 16 & 20 & $0 \cdot 40^{\mathrm{a}}$ \\
\hline Poultry & 15 & 13 & 1 & 0 & 0 & $-0 \cdot 14^{\mathrm{a}}$ \\
\hline Fish & 45 & 42 & 36 & 36 & 32 & $-0 \cdot 14^{\mathrm{a}}$ \\
\hline Potatoes & 72 & 85 & 92 & 98 & 110 & $0 \cdot 24^{\mathrm{a}}$ \\
\hline Fried & 0 & 0 & 0 & 0 & 0 & $0 \cdot 13^{\mathrm{a}}$ \\
\hline Boiled & 52 & 66 & 71 & 77 & 81 & $0 \cdot 19^{a}$ \\
\hline Cereals & 22 & 20 & 19 & 18 & 17 & $-0 \cdot 11^{\mathrm{a}}$ \\
\hline Vegetables & 141 & 130 & 128 & 136 & 132 & $-0 \cdot 05^{\mathrm{a}}$ \\
\hline Fruits & 220 & 212 & 204 & 200 & 184 & $-0 \cdot 11^{\mathrm{a}}$ \\
\hline Bread, white & 79 & 73 & 74 & 72 & 69 & $-0 \cdot 06^{\mathrm{a}}$ \\
\hline Bread, crisp & 19 & 19 & 17 & 17 & 15 & $-0 \cdot 08^{a}$ \\
\hline Egg & 17 & 19 & 19 & 19 & 20 & $0 \cdot 04$ \\
\hline Milk & 199 & 223 & 229 & 240 & 211 & $0 \cdot 02^{\mathrm{a}}$ \\
\hline Cream & 11 & 12 & 11 & 10 & 9 & $-0 \cdot 06^{\mathrm{a}}$ \\
\hline Cheese & 45 & 40 & 37 & 38 & 33 & $-0 \cdot 13^{a}$ \\
\hline Edible fats & 34 & 34 & 33 & 34 & 33 & $-0.02^{\mathrm{a}}$ \\
\hline Edible fat $100 \%$ & 0 & 0 & 0 & 0 & 0 & $0 \cdot 07^{\mathrm{a}}$ \\
\hline Edible fat $80 \%$ & 6 & 7 & 7 & 7 & 7 & $0 \cdot 04^{b}$ \\
\hline Edible fat $60 \%$ & 0 & 0 & 0 & 0 & 0 & $-0 \cdot 01$ \\
\hline Edible fat $40 \%$ & 9 & 12 & 12 & 12 & 11 & $0 \cdot 01$ \\
\hline Butter & 0 & 0 & 0 & 0 & 0 & $-0 \cdot 04^{\mathrm{b}}$ \\
\hline Mayonnaise/dressing & 2 & 3 & 2 & 2 & 2 & $-0 \cdot 01^{\mathrm{a}}$ \\
\hline Sweets & 14 & 15 & 15 & 15 & 14 & $-0 \cdot 01$ \\
\hline \multicolumn{7}{|l|}{ Alcoholic beverages } \\
\hline Hard liquor & 0 & 0 & 0 & 0 & 0 & $0 \cdot 02$ \\
\hline Wine & 21 & 21 & 17 & 14 & 29 & $-0 \cdot 01$ \\
\hline Beer & 0 & 14 & 14 & 14 & 0 & $0 \cdot 00$ \\
\hline
\end{tabular}

A significant trend between quintiles are given as Pearson correlation coefficients and a denotes $p<0 \cdot 001$ and $^{\mathrm{b}}=p<0 \cdot 01$.

protein, mono-unsaturated fat, palmitic acid (C 16:0), zinc and some vitamins like niacin and thiamin but lower intake of carbohydrates, ascorbic acid, fiber, lauric (C 12:0) and myristic acid (C 14:0), cobalamine, riboflavin and magnesium than low meat consumers, in both sexes. The female high consumers of meat had higher intake of total fat, saturated and nono-unsaturated fatty acids compared to women with low meat intake. The contribution of fat from meat out of the total fat intake was $11.9 \%$ for men and $13.6 \%$ for women. About $8 \%$ out of the total intake of polyunsaturated fat came from meat (Table 7).

A linear regression model between meat and single food groups showed that a $100 \mathrm{~g}$ increase of meat intake corresponded to a concurrent decrease of fruit intake 
TABLE 5

Daily intake of nutrients divided by quintiles of energy-adjusted meat consumption, in 4858 men

\begin{tabular}{|c|c|c|c|c|c|c|}
\hline Nutrient median & $\begin{array}{c}1 \\
\text { (Low) }\end{array}$ & 2 & 3 & 4 & $\begin{array}{c}5 \\
\text { (High) }\end{array}$ & $r$ \\
\hline Energy (kcal) & 2617 & 2613 & 2589 & 2603 & 2498 & $-0 \cdot 05^{\mathrm{a}}$ \\
\hline Protein (g) & 91 & 94 & 93 & 96 & 100 & $0 \cdot 11^{\mathrm{a}}$ \\
\hline Carbohydrates (g) & 295 & 289 & 275 & 271 & 252 & $-0 \cdot 19^{a}$ \\
\hline Alcohol (g) & $9 \cdot 9$ & $10 \cdot 5$ & $11 \cdot 2$ & $12 \cdot 6$ & $13 \cdot 5$ & $0.07^{\mathrm{a}}$ \\
\hline Fat (g) & 106 & 108 & 108 & 107 & 107 & $0 \cdot 02$ \\
\hline \multicolumn{7}{|l|}{ Saturated FA (g) } \\
\hline Total & 44 & 45 & 44 & 45 & 44 & $0 \cdot 00$ \\
\hline $12: 0$ & $2 \cdot 9$ & $2 \cdot 8$ & $2 \cdot 8$ & $2 \cdot 7$ & $2 \cdot 4$ & $-0 \cdot 12^{\mathrm{a}}$ \\
\hline $14: 0$ & $4 \cdot 8$ & $4 \cdot 6$ & $4 \cdot 6$ & $4 \cdot 5$ & $4 \cdot 3$ & $-0 \cdot 09^{a}$ \\
\hline $16: 0$ & $20 \cdot 8$ & $21 \cdot 4$ & $21 \cdot 4$ & $21 \cdot 9$ & $21 \cdot 6$ & $0 \cdot 04^{b}$ \\
\hline $18: 0$ & $9 \cdot 6$ & $10 \cdot 0$ & $10 \cdot 2$ & $10 \cdot 4$ & $10 \cdot 4$ & $0 \cdot 09^{\mathrm{a}}$ \\
\hline $20: 0$ & $0 \cdot 4$ & $0 \cdot 4$ & $0 \cdot 4$ & $0 \cdot 4$ & $0 \cdot 4$ & $-0.03^{c}$ \\
\hline \multicolumn{7}{|c|}{ Monosaturated FA (g) } \\
\hline Total & 36 & 38 & 38 & 38 & 39 & $0 \cdot 08^{\mathrm{a}}$ \\
\hline $16: 1$ & $2 \cdot 0$ & $2 \cdot 1$ & $2 \cdot 1$ & $2 \cdot 2$ & $2 \cdot 3$ & $0 \cdot 14^{\mathrm{a}}$ \\
\hline $18: 1$ & $32 \cdot 3$ & $33 \cdot 5$ & $34 \cdot 0$ & $34 \cdot 4$ & $34 \cdot 4$ & $0 \cdot 08^{\mathrm{a}}$ \\
\hline \multicolumn{7}{|c|}{ Polyunsaturated FA (g) } \\
\hline Total & 17 & 18 & 18 & 18 & 17 & $-0 \cdot 03$ \\
\hline $18: 2$ & $13 \cdot 8$ & $14 \cdot 3$ & $14 \cdot 1$ & $13 \cdot 8$ & $13 \cdot 5$ & $-0.03^{\mathrm{c}}$ \\
\hline $18: 3$ & $2 \cdot 0$ & $2 \cdot 1$ & $2 \cdot 1$ & $2 \cdot 0$ & $1 \cdot 9$ & $-0 \cdot 04^{\mathrm{b}}$ \\
\hline $20: 4$ & $0 \cdot 2$ & $0 \cdot 2$ & $0 \cdot 2$ & $0 \cdot 2$ & $0 \cdot 2$ & $0 \cdot 20^{\mathrm{a}}$ \\
\hline $20: 5$ & $0 \cdot 2$ & $0 \cdot 2$ & $0 \cdot 1$ & $0 \cdot 1$ & $0 \cdot 1$ & $-0 \cdot 11^{\mathrm{a}}$ \\
\hline $22: 5$ & $0 \cdot 1$ & $0 \cdot 1$ & $0 \cdot 1$ & $0 \cdot 1$ & $0 \cdot 1$ & $0 \cdot 18^{\mathrm{a}}$ \\
\hline $22: 6$ & $0 \cdot 4$ & $0 \cdot 4$ & $0 \cdot 3$ & $0 \cdot 3$ & $0 \cdot 3$ & $-0 \cdot 06^{\mathrm{a}}$ \\
\hline Zinc (mg) & 12 & $12 \cdot 7$ & $12 \cdot 7$ & $13 \cdot 3$ & $14 \cdot 0$ & $0 \cdot 19^{\mathrm{a}}$ \\
\hline Iron (mg) & $18 \cdot 5$ & $18 \cdot 5$ & $17 \cdot 9$ & $18 \cdot 1$ & $17 \cdot 9$ & $-0.05^{\mathrm{a}}$ \\
\hline Magnesium (mg) & 402 & 394 & 382 & 384 & 382 & $-0 \cdot 08^{a}$ \\
\hline Riboflavin (mg) & $2 \cdot 1$ & $2 \cdot 1$ & $2 \cdot 0$ & $2 \cdot 0$ & $2 \cdot 0$ & $-0 \cdot 07^{\mathrm{a}}$ \\
\hline Tiamin (mg) & $1 \cdot 5$ & $1 \cdot 6$ & $1 \cdot 5$ & $1 \cdot 6$ & $1 \cdot 6$ & $0 \cdot 03$ \\
\hline Niacin (mg) & $20 \cdot 2$ & $20 \cdot 3$ & $20 \cdot 2$ & $20 \cdot 9$ & $21 \cdot 7$ & $0 \cdot 11^{\mathrm{a}}$ \\
\hline Cobalamine $(\mu \mathrm{g})$ & $6 \cdot 9$ & $6 \cdot 8$ & $6 \cdot 5$ & $6 \cdot 7$ & $6 \cdot 5$ & $-0 \cdot 04^{b}$ \\
\hline Tocopherol (mg) & $9 \cdot 8$ & $9 \cdot 9$ & $9 \cdot 6$ & $9 \cdot 4$ & $9 \cdot 4$ & $-0 \cdot 07^{\mathrm{a}}$ \\
\hline Ascorbic acid (mg) & $95 \cdot 2$ & $90 \cdot 0$ & $83 \cdot 7$ & $84 \cdot 3$ & $81 \cdot 9$ & $-0 \cdot 11^{\mathrm{a}}$ \\
\hline Retinol $(\mu \mathrm{g})$ & 1264 & 1375 & 1256 & 1247 & 1118 & $-0 \cdot 06^{\mathrm{a}}$ \\
\hline Carotene $(\mu \mathrm{g})$ & 2682 & 2321 & 2320 & 2127 & 2254 & $-0 \cdot 07^{\mathrm{a}}$ \\
\hline Fiber (g) & $23 \cdot 7$ & $22 \cdot 8$ & $21 \cdot 0$ & $21 \cdot 2$ & $19 \cdot 8$ & $-0 \cdot 20^{\mathrm{a}}$ \\
\hline
\end{tabular}

$\mathrm{FA}=$ fatty acids

A significant trend between quintiles are presented and ${ }^{\mathrm{a}}$ denotes $p<0 \cdot 001,{ }^{\mathrm{b}}=p<0 \cdot 01$ and ${ }^{\mathrm{c}}=$ $p<0 \cdot 05$.

of approximately $15-18 \mathrm{~g}$ in men and women, whereas the intake of milk would increase with $33-51 \mathrm{~g}$ and edible fats less than $7 \mathrm{~g}$ in both sexes (Table 8).

\section{DisCUSSION}

In this study, intake of fat from meat constituted approximately $13 \%$ of total fat intake. Other more important sources of fat were dairy products, and edible fats. 
TABLE 6

Daily intake of nutrients divided by quintiles of energy-adjusted meat consumption, in 6788 women

\begin{tabular}{|c|c|c|c|c|c|c|}
\hline Nutrient median & $\begin{array}{c}1 \\
\text { (Low) }\end{array}$ & 2 & 3 & 4 & $\begin{array}{c}5 \\
\text { (High) }\end{array}$ & $r$ \\
\hline Energy (kcal) & 1984 & 1987 & 1961 & 1993 & 1997 & $0 \cdot 00$ \\
\hline Protein (g) & 73 & 75 & 75 & 78 & 80 & $0 \cdot 13^{\mathrm{a}}$ \\
\hline Carbohydrates (g) & 229 & 224 & 220 & 220 & 209 & $-0 \cdot 10^{\mathrm{a}}$ \\
\hline Alcohol (g) & $5 \cdot 3$ & $5 \cdot 3$ & $5 \cdot 0$ & $4 \cdot 8$ & $5 \cdot 8$ & $0 \cdot 01$ \\
\hline Fat (g) & 79 & 80 & 81 & 82 & 84 & $0 \cdot 06^{\mathrm{a}}$ \\
\hline \multicolumn{7}{|l|}{ Saturated FA (g) } \\
\hline Total & 34 & 34 & 34 & 35 & 36 & $0 \cdot 04^{\mathrm{b}}$ \\
\hline $12: 0$ & $2 \cdot 1$ & $2 \cdot 1$ & $2 \cdot 1$ & $2 \cdot 1$ & $2 \cdot 0$ & $-0 \cdot 06^{\mathrm{a}}$ \\
\hline $14: 0$ & $3 \cdot 8$ & $3 \cdot 7$ & $3 \cdot 6$ & $3 \cdot 6$ & $3 \cdot 6$ & $-0 \cdot 06^{\mathrm{a}}$ \\
\hline $16: 0$ & $15 \cdot 9$ & $16 \cdot 2$ & $16 \cdot 1$ & $16 \cdot 6$ & $17 \cdot 4$ & $0 \cdot 07^{\mathrm{a}}$ \\
\hline $18: 0$ & $7 \cdot 2$ & $7 \cdot 5$ & $7 \cdot 6$ & $7 \cdot 9$ & $8 \cdot 2$ & $0 \cdot 13^{\mathrm{a}}$ \\
\hline $20: 0$ & $0 \cdot 4$ & $0 \cdot 4$ & $0 \cdot 4$ & $0 \cdot 4$ & $0 \cdot 4$ & $-0 \cdot 01$ \\
\hline \multicolumn{7}{|c|}{ Monosaturated FA (g) } \\
\hline Total & 27 & 28 & 28 & 29 & 30 & $0 \cdot 12^{\mathrm{a}}$ \\
\hline $16: 1$ & $1 \cdot 5$ & $1 \cdot 6$ & $1 \cdot 6$ & $1 \cdot 7$ & $1 \cdot 8$ & $0 \cdot 15^{\mathrm{a}}$ \\
\hline $18: 1$ & $23 \cdot 7$ & $24 \cdot 7$ & $24 \cdot 9$ & $25 \cdot 4$ & $26 \cdot 8$ & $0 \cdot 12^{\mathrm{a}}$ \\
\hline \multicolumn{7}{|c|}{ Polyunsaturated FA (g) } \\
\hline Total & 13 & 13 & 13 & 13 & 13 & $0 \cdot 01$ \\
\hline $18: 2$ & $10 \cdot 1$ & $10 \cdot 2$ & $10 \cdot 0$ & $10 \cdot 2$ & $10 \cdot 1$ & $0 \cdot 01$ \\
\hline $18: 3$ & $1 \cdot 5$ & $1 \cdot 5$ & $1 \cdot 5$ & $1 \cdot 5$ & $1 \cdot 5$ & $0 \cdot 00$ \\
\hline $20: 4$ & $0 \cdot 1$ & $0 \cdot 1$ & $0 \cdot 1$ & $0 \cdot 1$ & $0 \cdot 2$ & $0 \cdot 23^{\mathrm{a}}$ \\
\hline $20: 5$ & $0 \cdot 1$ & $0 \cdot 1$ & $0 \cdot 1$ & $0 \cdot 1$ & $0 \cdot 1$ & $-0 \cdot 09^{a}$ \\
\hline $22: 5$ & $0 \cdot 0$ & $0 \cdot 1$ & $0 \cdot 1$ & $0 \cdot 1$ & $0 \cdot 1$ & $0 \cdot 22^{\mathrm{a}}$ \\
\hline $22: 6$ & $0 \cdot 3$ & $0 \cdot 3$ & $0 \cdot 2$ & $0 \cdot 2$ & $0 \cdot 2$ & $-0 \cdot 05^{\mathrm{a}}$ \\
\hline Zinc (mg) & $9 \cdot 3$ & $9 \cdot 3$ & $9 \cdot 8$ & $10 \cdot 5$ & $11 \cdot 0$ & $0 \cdot 22^{\mathrm{a}}$ \\
\hline Iron (mg) & $13 \cdot 5$ & $13 \cdot 5$ & $13 \cdot 3$ & $13 \cdot 5$ & $13 \cdot 7$ & $0 \cdot 02$ \\
\hline Magnesium (mg) & 328 & 321 & 315 & 320 & 314 & $-0 \cdot 04^{\mathrm{a}}$ \\
\hline Riboflavin (mg) & $1 \cdot 7$ & $1 \cdot 7$ & $1 \cdot 7$ & $1 \cdot 7$ & $1 \cdot 7$ & $-0.03^{c}$ \\
\hline Tiamin (mg) & $1 \cdot 2$ & $1 \cdot 2$ & $1 \cdot 2$ & $1 \cdot 3$ & $1 \cdot 3$ & $0 \cdot 10^{\mathrm{a}}$ \\
\hline Niacin (mg) & $15 \cdot 1$ & $15 \cdot 4$ & $15 \cdot 7$ & $16 \cdot 1$ & $16 \cdot 9$ & $0 \cdot 15^{\mathrm{a}}$ \\
\hline Cobalamine $(\mu \mathrm{g})$ & $5 \cdot 2$ & $5 \cdot 1$ & $5 \cdot 1$ & $5 \cdot 2$ & $5 \cdot 1$ & $-0 \cdot 01$ \\
\hline Tocopherol (mg) & $8 \cdot 6$ & $8 \cdot 3$ & $8 \cdot 1$ & $8 \cdot 2$ & $8 \cdot 0$ & $-0 \cdot 08^{a}$ \\
\hline Ascorbic acid (mg) & $113 \cdot 3$ & $107 \cdot 0$ & $103 \cdot 8$ & $101 \cdot 4$ & $94 \cdot 9$ & $-0 \cdot 11^{\mathrm{a}}$ \\
\hline Retinol $(\mu \mathrm{g})$ & 860 & 876 & 907 & 885 & 829 & $-0.03^{\mathrm{c}}$ \\
\hline Carotene $(\mu \mathrm{g})$ & 3544 & 3061 & 2970 & 3104 & 2822 & $-0 \cdot 09^{a}$ \\
\hline Fiber (g) & $21 \cdot 4$ & $19 \cdot 7$ & $19 \cdot 1$ & $19 \cdot 1$ & $19 \cdot 9$ & $-0 \cdot 16^{\mathrm{a}}$ \\
\hline
\end{tabular}

$\mathrm{FA}=$ fatty acids

A significant trend between quintiles are presented and ${ }^{\mathrm{a}}$ denotes $p<0 \cdot 001,{ }^{\mathrm{b}}=p<0 \cdot 01$ and ${ }^{\mathrm{c}}=$ $p<0 \cdot 05$.

Only $11 \cdot 6-13 \cdot 6 \%$ of saturated fat came from meat intake and since meat has lower proportion of polyunsaturated fatty acids (PUFA), the corresponding amount of PUFA from meat was about $8 \%$. The assessed intake of meat was similar to a previous Swedish nation-wide study of dietary habits reporting mean intake of meat, including poultry, approximately $97 \mathrm{~g}$ for men and $71 \mathrm{~g}$ for women compared to median intake of $104 \mathrm{~g}$ in men and $70 \mathrm{~g}$ in women in this study (Becker, 1992). A previous validity study with an 18-day dietary record as a reference, showed that 
TABLE 7

Mean proportion of total nutrient intake from mean intake of meat

\begin{tabular}{lrc}
\hline Percent nutrient from total meat & Men $(\%)$ & Women $(\%)$ \\
\hline Energy & $8 \cdot 2$ & $7 \cdot 1$ \\
Protein & $19 \cdot 7$ & $16 \cdot 6$ \\
Carbohydrates & $0 \cdot 5$ & $0 \cdot 4$ \\
Fat & $13 \cdot 6$ & $11 \cdot 9$ \\
$\quad$ Saturated fat & $13 \cdot 5$ & $11 \cdot 6$ \\
Monounsaturated fat & $17 \cdot 1$ & $15 \cdot 2$ \\
Polyunsaturated fat & $8 \cdot 3$ & $7 \cdot 4$ \\
Selenium & $14 \cdot 7$ & $12 \cdot 0$ \\
Zinc & $24 \cdot 6$ & $21 \cdot 3$ \\
Iron & $9 \cdot 9$ & $8 \cdot 7$ \\
Magnesium & $5 \cdot 9$ & $4 \cdot 8$ \\
Riboflavin & $9 \cdot 5$ & $7 \cdot 7$ \\
Tiamin & $14 \cdot 2$ & $12 \cdot 4$ \\
Niacin & $16 \cdot 3$ & $14 \cdot 1$ \\
Cobalamine & $14 \cdot 2$ & $11 \cdot 9$ \\
Tocopherol & $4 \cdot 2$ & $3 \cdot 3$ \\
\hline
\end{tabular}

men tended to overestimate meat intake in the order of $15 \%$, whereas women neither over- or underestimated meat consumption (Elmståhl et al., 1996a,b). Our results show that the overall consumption of meat among a Swedish population is low compared to other European countries. The per capita consumption of meat has increased more slowly in Sweden than in most other European countries over the past 30 years, from $51 \mathrm{~kg}$ per person per year in 1961 to $60 \mathrm{~kg}$ in 1990 compared to France (73-95 kg of meat), Italy (31-85 kg) and Switzerland (56-83 kg) (Food and Health Indicators, 1995).

Relationships between several dietary factors and certain types of cancer have been suggested in previous epidemiological studies. The associations between dietary fat and red meat, and colon cancer have been restricted to saturated and monounsaturated fat from animal sources, but not from vegetable fat (Willett et al., 1990). Recent studies have suggested different roles of fatty acids from the $\omega 3$ and $\omega 6$ series with an increased tumour growth by unsaturated fatty acids from the $\omega 6$ series (Carter et al., 1983; Endres et al., 1989; Karmali, 1987). Some of the inconclusive data regarding cancer incidence and meat consumption might be explained by differences in dietary habits, supported by the fact that only a minor part of fat and saturated fat comes from meat in a Scandinavian diet, as shown in this study. Furthermore, definitions of meat intake might differ between studies and the term "red meat" might include both processed meat (e.g. in sausage) and whole meat with different amounts of fat. Also other studies mainly include meat from dishes (Goldbohm et al., 1994; Willett et al., 1990). This might represent subpopulations with different dietary habits, a supposition also supported by the low correlation between intake of whole meat and processed meat $(-0.03$ to $0 \cdot 11)$, noted in this study.

Although a positive association was noted between the intake of potatoes, an important source of ascorbic acid, and meat, the overall correlations between ascorbic acid and fiber and meat were negative. It was found that several foods regarded as 
TABle 8

Linear regression with changes of food groups in gram and standard error (SE) per one $g$ meat intake

\begin{tabular}{|c|c|c|c|c|c|c|}
\hline & \multicolumn{3}{|c|}{ Men } & \multicolumn{3}{|c|}{ Women } \\
\hline & Change & $(S E)$ & Squared R & Change & $(S E)$ & Squared R \\
\hline Fish & $-0 \cdot 04$ & $(0 \cdot 010)$ & $0 \cdot 004$ & $-0 \cdot 07$ & $(0 \cdot 009)$ & 0.007 \\
\hline Potatoes & $0 \cdot 56$ & $(0.020)$ & $0 \cdot 141$ & $0 \cdot 47$ & $(0 \cdot 166)$ & $0 \cdot 106$ \\
\hline Vegetables & $0 \cdot 07$ & $(0.021)$ & 0.003 & $0 \cdot 04$ & $(0 \cdot 026)$ & $0 \cdot 000$ \\
\hline Fruits & $-0 \cdot 15$ & $(0.030)$ & $0 \cdot 005$ & $-0 \cdot 18$ & $(0 \cdot 037)$ & $0 \cdot 003$ \\
\hline Bread & $0 \cdot 10$ & $(0.021)$ & $0 \cdot 005$ & 0.08 & $(0 \cdot 016)$ & 0.003 \\
\hline Milk & $0 \cdot 33$ & $(0 \cdot 065)$ & $0 \cdot 001$ & $0 \cdot 51$ & $(0 \cdot 062)$ & $0 \cdot 010$ \\
\hline Cheese & $-0 \cdot 01$ & $(0 \cdot 008)$ & $0 \cdot 000$ & $-0 \cdot 03$ & $(0 \cdot 010)$ & $0 \cdot 001$ \\
\hline Edible fat & $0 \cdot 07$ & $(0.007)$ & 0.022 & $0 \cdot 06$ & $(0 \cdot 006)$ & $0 \cdot 016$ \\
\hline
\end{tabular}


"protective" with respect to oxidative burden decreased with higher intake of meat, e.g. cereals, fruits and bread. A similar pattern was noted in the Womens' Health study where women with a high intake of animal fat tended to consume less fiber (Willett et al., 1990). The risk of colon cancer increased when women with high fat/ low fiber intake were compared with low fat/high fiber intake, but the difference in total fiber intake between highest and lowest quintile, $9 \cdot 7 \mathrm{~g}$ daily, was greater than our data. Meat is an important source of zinc, selenium and iron in a Scandinavian diet. Previous observations have indicated a cancer promoting effect by body iron, maybe through catalysing production of oxygen radicals. In a prospective cohort study of men, those who developed cancer had higher transferrin saturation 4 years before diagnosis than men who did not develop cancer (Sciemeca \& Thompson, 1994). However, in this study a weak negative association between the intake of iron and meat was found in men. As bread intake decreases with higher meat intake the non-haeme iron will be replaced by haeme-iron with higher bioavailability. About $90 \%$ of the total iron intake constituted non-haeme iron and this would correspond to about $80 \%$ of total iron considering the higher absorption of haeme iron (Stevens, 1990). In Sweden, the contribution of iron from meat has remained roughly the same, approximately $3 \mathrm{mg}$, over the last 20 years whereas a decrease of iron from bread can be foreseen since the iron fortification of flour was stopped 1995 (Hallberg, 1981). An increase of iron deficiency might be anticipated in subjects with dietary habits characterized by low intakes of vegetables and meat compared to other European countries.

In this study, dietary habits differed between high and low consumers of meat. High consumers reported lower intakes of bread, cereals, fruits, fish including seafood, fat from animal sources like milk, cheese and cream, and edible fats with high amounts of vegetable fats. Since fruit and vegetables seem to protect against cancer, a negative relationship between intake of meat and fruit will underestimate any potential risk effect of fat if not including fruit in analyses. Previous diet and colon cancer studies have reported a decreased risk with higher intake of PUFA and seafood, and an increased risk with higher intake of sucrose containing foods, saturated fats and (in some studies) meat (Bostick et al., 1994; Willett et al., 1990). Data on meat and colon cancer relationships from more than 20 studies show a positive association in more than half of the studies and no association in the others, with one exception (Bostick et al., 1994). Some of the differences between these studies might be explained by different study design, study populations, sample sizes and diet assessment methods with varying ability to cover heterogeneity in diet. Some large cohort studies have not been able to show association between the intake of meat and vegetables/fiber and colon cancer (Bostick et al., 1994; Goldbohm et al., 1994). However, a collinearity between food items, with potentially different effects on disease outcome, might influence the observed relative risk which has been described earlier for linear regression models (Elmståhl \& Gullberg, 1997). Assuming a model with two risk factors, $\mathrm{RR}=2 \cdot 0$ and $\mathrm{RR}=0.5$; a validity of about 0.7 to assess the risk factors compared to a reference method and a collinearity in the order of $0 \cdot 3$, comparable to e.g. the correlation between potatoes and meat in this study, the observed RR would then be approximately $1 \cdot 3$. However, this kind of measurement error should be limited since the collinearity between several food groups like vegetables, potatoes and fruits and meat was low with squared $\mathrm{R}$ values below 0.10 in most cases. The food frequency questionnaire, a part of the dietary assessment method, covered the past year. Seasonal intake of fruit could, therefore, 
be taken into consideration and included in the mean calculated intakes of food groups. The linear regression model might indicate a cluster of food habits in relation to meat intake.

Men reported higher intake of hard liquor and total intake of alcohol with increasing meat intake. Case-control and cohort studies have reported an association between alcoholic beverages and large-bowel cancer, especially rectal cancer (Blot, 1992; Stemmermann et al., 1984). In a Japanese cohort study, the relative risk of daily vs. non-daily alcohol consumption for cancer of the sigmoid colon was $2 \cdot 1$, adjusted for diet and smoking (Hirayama, 1989). Other studies have reported an increased risk for rectal cancer and alcohol, especially for beer drinkers (Kabat et al., 1986; Miller et al., 1983).

Colorectal cancer has been associated with a "Western" type of diet rich in animal foods and fat and a low content of fiber (Burkitt, 1969). The incidence of colorectal cancer in Sweden over the last 20 years has shown an annual increase of 0.5-1\% (Cancer Incidence in Sweden 1990, 1993). On the other hand, colorectal mortality in Sweden has decreased by around the same since 1974 (Causes of Death, 1989). This might be compatible with earlier diagnosis and improved treatment but the influence of changed dietary habits and environmental factors are difficult to estimate. The same decline of colorectal cancer mortality has been observed in many other countries where meat consumption has increased indicating that other factors may be of importance.

A link has been postulated between cardiovascular disease, atherosclerosis and dietary factors. Some saturated fatty acids like lauric, myristic and palmitic acids have been suggested as atherogenic fatty acids (Keys et al., 1965), whereas some PUFAs are believed to have antithrombogenic activity (Sinclair et al., 1994). Data on meat consumption and coronary heart disease have been conflicting. In this study, high consumers of meat had about the same intake of the suggested atherogenic fatty acids as low consumers ( $28.3 \mathrm{~g}$ vs. $28.5 \mathrm{~g})$. Neither did we find increased meat intake to be associated with an increase of arachidonic acid or linoleic acid, the major precursor of arachidonic acid which has been suspected to promote thrombosis (Sinclair et al., 1994). Another PUFA such as C:20:503 - a precursor of prostaglandin $\mathrm{E}_{1}$ with a proposed antithrombotic effect-showed a weak negative association with meat intake. A balanced diet of the different PUFAs might, therefore, be preferable due to indications of different antagonizing biological effects to reduce suggested atherogenic effects.

Thus, it seems that a meat containing diet can harbour both potentially carcinogenic and anticarcinogenic substances as well as atherogenic substances. The summation of these effects may be difficult to disentangle from the effects of other food items due to the problem of collinearity. Fat derived from meat only constitutes a minor part of the total fat intake in a Scandinavian diet. Large cohort studies are needed to analyse the relationships between meat/fat and cancer due to confounding by several dietary and non-dietary factors.

\section{REFERENCES}

Armstrong, B. K. \& Doll, R. (1975). Environmental factors and cancer incidence and mortality in different countries, with special reference to dietary practices. International Journal of Cancer, 15, 617-631. 
Balla, G., Jacob, H. S., Eaton, J. W., Belcher, J. D., Vercellotil, G. M. \& Hemin A. (1991). A possible physiological mediator of low density lipoprotein oxidation and endothelial injury. Arteriosclerosis and Thrombosis, 11, 1700-1711.

Becker, W. (1992). Food habits and nutrient intake in Sweden. (1989). National Food Administration Vår Föda, 8, 349-362.

Berglund, G., Elmståhl, S., Janzon, L. \& Larsson, A. (1993). The Malmö Diet and Cancer Study. Design and feasibility. Journal of Internal Medicine, 233, 45-51.

Blot, W. (1992). Alcohol and cancer. Cancer Research, 52, 2119S-2123S.

Bostick, R., Potter, J., Kushi, L., Sellers, A., Steinmetz, D. R., McKenzie, D. R., Gapstur, S. M. \& Folsom, A. R. (1994). Sugar, meat, and fat intake and non-dietary risk factors for colon cancer incidence in Iowa women (United States). Cancer Causes and Control, $5,38-52$.

Burkitt, D. P. (1969). Related disease-related cause? Lancet, 2, 1229-1231.

Cancer Incidence in Sweden 1990. (1993). The National Board of Health and Welfare. Centre for Epidemiology. The Swedish Cancer Registry. Stockholm.

Carrol, K. K. (1975). Experimental evidence of dietary factors and hormone-dependent cancers. Cancer Research, 33, 3374-3383.

Carter, C., Milholland, R. J. \& Shea W. (1983). Effect of the prostaglandin synthetase inhibitor indomethacin on 7,12-dimetylbenz $(\alpha)$ anthracen-induced mammary tumorigenesis in rats fed different levels of fat. Cancer Research, 43, 3559-3562.

Causes of Death 1989. (1992). Official Statistics of Sweden. National Central Bureau of Statistics. Stockholm.

Elmståhl, S. \& Gullberg, B. (1997). Bias in diet assessment methods-consequences of collinearity on sample size calculations and exposure variables. International Journal of Epidemiology, 26, 1071-1079.

Elmståhl, S., Wallström, P., Berglund, G., Janzon, L., Johansson, U., Larsson, A. \& Mattisson, I. (1994). The use of dietary supplements in relation to dietary habits in a Swedish middleaged population. Scandinavian Journal of Nutrition, 38, 94-97.

Elmståhl, S., Riboli, E., Lindgärde, F., Gullberg, B. \& Saracci, R. (1996a). The Malmö Food Study. Validity of a modified diet history method and an extensive food frequency questionnaire for measuring food intake. European Journal of Clinical Nutrition, 50, $143-151$.

Elmståhl, S., Gullberg, B., Riboli, E., Saracci, R. \& Lindgärde, F. (1996b). The Malmö Food Study. The reproducibility of a novel diet history method and an extensive food frequency questionnaire. European Journal of Clinical Nutrition, 50, 134-142.

Endres, S., Ghorbani, R. \& Kelley, V. E. (1989). The effect of dietary supplementation with n-3 polyunsaturated fatty acids on the synthesis of interleukin-1 and tumor necrosis factor by mononuclear cells. New England Journal of Medicine, 320, 265-271.

Ewertz, M. \& Gill, C. (1990). Dietary factors and breast cancer risk in Denmark. International Journal of Cancer, 46, 779-784.

Food and Health Indicators (1995). Nutrition Programme. WHO Regional Office for Europe. version 3. Copenhagen.

Galli, C., Mosconi, C., Medini, L. \& Tremoli, E. (1989). Dietary fatty acids, serum lipids, platelet biochemistry and function. Wiener Klinische Wochenzeitschrift, 14, 267-271.

Giovanucci, E., Rimm, E. B., Colditz, G. A., Stampfer, M. J., Ascherio, A., Chute, C. C. \& Willett, W. C. (1993). A prospective study of dietary fat and risk of prostate cancer. Journal of National Cancer Institute, 85, 1571-1579.

Giovanucci, E., Rimm, E. B., Stampfer, M. J., Colditz, G. A., Ascherio, A. \& Willett, W. C. (1994). Intake of fat, meat and fiber in relation to risk of colon cancer in man. Cancer Research, 54, 2390-2397.

Goldbohm, A., van den Brandt, P., van't Veer, P., Brants, H., Dorant, E., Sturmans, F. \& Hermus, R. (1994). A prospective cohort study on the relation between meat consumption and the risk of colon cancer. Cancer Research, 54, 718-723.

Gotto, A. M., LaRosa, J. C., Hunninghake, D., Grundy, S. M., Wilson, P. W., Clarkson, T. B. \& Goodman, D. W. S. (1990). The cholesterol facts. A summary relating dietary fats, serum cholesterol and coronary heart disease. Circulation, 81, 1721-1733.

Hallberg, L. (1981). Bioavailability of dietary iron in man. Annual Review of Nutrition, 1, 123-147. 
Hill, P., Wynder, E. L. \& Grabaczewski, E. (1987). Diet and urinary steroids in black and white North American men. Cancer Research, 47, 2982-2985.

Hirayama, T. (1981). A large-scale cohort study on the relationship between diet and selected cancers of digestive organs. In W. Bruce, P. Correa, M. Lipkin, S. R. Tannenbaum \& T. D. Wilkins (Eds) Gastrointestinal Cancer. Endogenous factors, pp. 409-426. Banbury Report 7. New York: Cold Spring Harbor Laboratory.

Hirayama, T. (1989). Association between alcohol consumption and cancer of the sigmoid colon: Observations from a Japanese cohort study. Lancet, 23, 725-727.

Kabat, C. G., Howson, C. P. \& Wynder, E. L. (1986). Beer consumption and rectal cancer. International Journal of Epidemiology, 15, 454-501.

Karmali, R. A. (1987). Omega-3 FA and cancer: A review. In W. E. M. Lands (Ed.) Polyunsaturated fatty acids and eicosanoids, pp. 222-233. Champaign, AOCS.

Keys, A., Anderson, J. T. \& Grande, F. (1965). Serum cholesterol response to changes in the diet. IV. Particularly saturated fatty acids in the diet. Metabolism, 14, 776-787.

Leaf, A. \& Weber, P. C. (1988). Cardiovascular effects of n-3 fatty acids. New England Journal of Medicine, 318, 549-557.

Miller, A. B., Howe, G. R., Jain, M., Crain, K. J. P., \& Harrison, L. (1983). Food items and food groups as risk factors in case-control study of diet and colo-rectal cancer. International Journal of Cancer, 32, 155-161.

Philips, R. \& Snowdon, D. (1985). Dietary relationships with fatal colorectal cancer among Seven-Day Adventists. Journal of National Cancer Institute, 74, 307-317.

Potter, J. D. (1996). Nutrition and colorectal cancer. Cancer Causes Control, 7, 127-146.

Riboli, E., Elmståhl, S., Saracci, R., Lindgärde, F. \& Gullberg, B. (1997). The Malmö Food Study. Validity of two dietary assessment methods for measuring usual nutrient intake. International Journal of Epidemiology, 26, S161-S173.

Sciemeca, J. A. \& Thompson, H. J. (1994). Conjugated linoleic acid. A powerful anticarcinogen from animal fat sources. Cancer, 74, S1050-S1054.

Sinclair, A., Johnson, L., O’Dea, K. \& Holman, R. (1994). Diets rich in lean beef increase arachidonic acid and long-chain $\omega 3$ polyunsaturated fatty acid levels in plasma phospholipids. Lipids, 29, 337-343.

Stemmermann, G., Nomura, A. \& Heilbrun, L. (1984). Dietary fat and the risk of colorectal cancer. Cancer Research, 44, 4633-4637.

Stevens, R. G. (1990). Iron and the risk of cancer. Medical Oncology \& Tumor Pharmacology, 7, 177-181.

Ulbricht, T. \& Southgate, D. (1991). Coronary heart disease: seven dietary factors. Lancet, 338, 985-992.

Willett, W., Stampfer, M., Colditz, G., Rossner, B. A. \& Speizer, F. E. (1990). Relation of meat, fat and fiber intake to the risk of colon cancer in a prospective study among women. New England Journal of Medicine, 323, 1164-1172.

Received 15 September 1997, revision 9 April 1998, accepted in revised form 12 May 1998 